$$
N 84-23991
$$

Nons

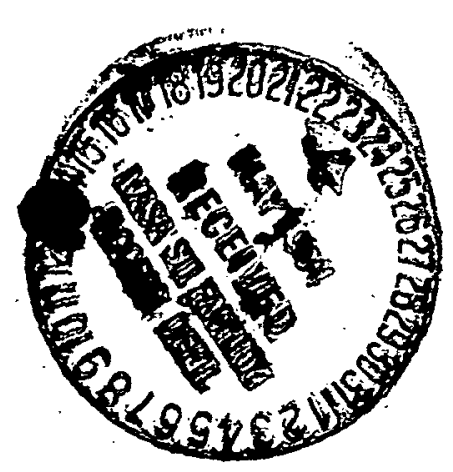

Technical Memorandum 86095

\title{
PREDICTABILITY OF THE EARTH'S POLAR MOTION
}

\section{B. Fong Chao}

\section{APRIL 1984}

\author{
National Aeronautics and \\ Space Administration
}

Goddard Space Flight Center

Greenbelt. Maryland 20771 


\title{
PREDICTABILITY OF THE EARTH'S POLAR MOTION
}

\author{
B. Fong Chao
}

\section{GODDARD SPACE FLIGHT CENTER}

Greenbelt, Maryland 


\section{Page intentionally left blank}

\section{Page intentionally left blank}




\title{
PREDICTABILITY OF THE EARTH'S POLAR MOTION \\ B. Fong Chao
}

\begin{abstract}
The numerical prediction of the Earth's polar motion is of both theoretical and practical interests. The present paper is aimed at a comprehensive, experimental study of the predictability of the polar motion using a homogeneous BIH (Bureau International de l'Heure) data set for the period 1967-1983. Based on our knowledge of the physics of the annual and the Chandler wobbles, we build the numerical model for the polar motion by allowing the wobble periods to vary. Using an optimum base length of 6 years for prediction, this "floating-period" model, equipped with a non-linear least-squares estimator, is found to yield polar motion predictions accurate to within $0^{\prime \prime} .012$ to $0 " .024$ depending on the prediction length up to one year, corresponding to a predictability of $91-83 \%$. This represents a considerable improvement over the conventional fixed-period predictor, which, by its nature, does not respond to variations in the apparent wobble periods (in particular, a dramatic decrease in the periods of both the annual and the Chandler wobbles after the year 1980). The superiority of the floating-period predictor to other predictors based on critically different numerical models is also demonstrated.
\end{abstract}




\section{PREDICTABILITY OF THE EARTH'S POLAR MOTION}

\section{INTRODUCTION}

The art of prediction of a time series consists in the extrapolation into the future of past behavior of the dynamic system that generates the time series. Some systems are deterministic and hence completely predictable (at least in principle), such as tides or the time of the next sunrise. Other systems are for the most part statistical in nature and often too erratic to be subjected to accurate predictions; examples include the weather pattern and stock market tradings.

The Earth's polar motion is a dynamic system that lies somewhere between the two extremes (a quantitative treatment is given in Section 4). To first approximation, the polar motion is composed of a secular motion and two quasi-circular components: the annual wobble and the 14-month Chandler wobble. These components are by no means completely deterministic (as are, say, the tides), yet each of them, governed by different physics, is coherent enough with respect to time that a fairly accurate prediction can be expected. This makes the problem of polar motion prediction meaningful and, in my opinion, challenging.

Besides theoretical interests, the prediction of polar motion is of practical value as well, as attested by the independent publication of predicted pole positions by two time services: the BIH (Bureau International de l'Heure) Circular B/C [Feissel, 1980], and the USNO (U.S. Naval Observatory) Series 7 [Babcock, 1982]. The conventional prediction method adopted is one that can be characterized by fixed wobble periods. Zhu [1982] has made a comprehensive study of polar motion prediction that uses such a "fixed-period" scheme; it, as far as I know, has remained the only published work on the subject to date.

The present paper is an experimental study of the predictability of the polar motion based on a homogeneous BIH data set for the period 1967-1983. It will be shown that with a floatingperiod numerical model and a capable estimation method. predictions accurate to within $0 " .012$ to $0 " .024$ can be achieved for the polar motion, depending on the prediction length up to one year. 
The superiority of this "floating-period predictor" to other predictors that are based on critically different numerical models (including the conventional fixed-period predictor) will be demonstrated.

\section{PREDICTION (using the Floating-Period Predictor)}

The procedure of numerical prediction consists of three stages: model identification, parameter estimation, and extrapolation [Box \& Jenkins, 1970].

\subsection{Model Identification}

Based on our knowledge of the physics of the polar motion, we decompose the latter into three major components:

$$
\text { polar motion }=(\text { secular motion })+(\text { annual wobble })+(\text { Chandler wobble })
$$

For the purpose of prediction, we shall consider record segments with lengths shorter than, say, 8 years. It is with respect to this time scale that we discuss the numerical representation of each component in equation (1).

The secular motion (relative to the Conventional International Origin, or $\mathrm{CIO}$ ) can be wellrepresented by a linear term. It is presumably the combination of a true polar wander and any periodic component with a period much longer than, say, 8 years.

The annual wobble is believed to be driven largely by the variations in the atmospheric mass distribution [see e.g. Lambeck, 1980]. The solar energy received by the atmosphere cycles every 365.25 days with a nearly constant peak-to-peak amplitude. However, the Earth's meteorological transfer function being intricate and non-linear, it is only natural that the annual wobble thereby generated will have an amplitude that varies from year to year and a period that fluctuates about the driving period 365.25 days.

The Chandler wobble is the Earth's free oscillation that corresponds to the Eulerian nutation of a rigid body; but the exact physical nature of its variations with time is largely unknown. One 
hypothesis states that the Chandler wobble is simply a single-period oscillation convolved with a sequence of random or non-random excitations. An alternative hypothesis calls for multiple (and fixed) natural periods [see.e.g. Colombo \& Shapiro, 1968]. Both hypotheses give rise to (apparent) instantaneous periods and amplitudes that are slowly varying with respect to time, as manifested by actual observations. (For the single-component hypothesis, a good synthetic example is given by the "disturbed pendulum" model of Yule [1927]; for the modulating effects of multiple periods on the instantaneous period and amplitude of the polar motion, see Chao [1983] .)

The above discussions justify the following numerical model for the polar motion:

$$
\mathrm{X}(\mathrm{t})=\mathrm{A}+\mathrm{Bt}+\mathrm{C}_{\mathrm{a}} \cos \left(\frac{2 \pi \mathrm{t}}{\mathrm{P}_{\mathrm{a}}}+\phi_{\mathrm{a}}\right)+\mathrm{C}_{\mathrm{c}} \cos \left(\frac{2 \pi \mathrm{t}}{\mathrm{P}_{\mathrm{c}}}+\phi_{\mathrm{c}}\right)
$$

for the $\mathrm{X}$-component, and a similar expression for the $\mathrm{Y}$-component $\mathrm{Y}(\mathrm{t})$. A+Bt is the linear secular term, and the two sinusoidal terms represent the annual and the Chandler wobbles ( $\mathrm{C}$ stands for the amplitude, $\mathrm{P}$ the period, and $\phi$ the phase angle). The eight (real) parameters $A, B, C_{a}, P_{a}, \phi_{a}, C_{c}$, $P_{C}, \phi_{C}$ are unknowns to be estimated from a given record segment $X(t)$ or $Y(t)$ - each record segment yields one set of estimated parameters. Since we allow the data in each given record segment to "choose" the wobble periods, we call equation (2) the floating-period model. Note that here we treat $X(t)$ and $Y(t)$ as independent series so as not to restrict ourselves only to the prograde portion of the true polar motion, as would if we chose to model the complex series $X(t)+i Y(t)$ in terms of "complex wobbles" of the form $\exp (i 2 \pi t / P+\phi)$. Although the physics behind equation (2) is for the most part unknown, we shall show that this model is adequate for the purpose of prediction.

\subsection{Parameter Estimation Method}

The next stage is, of course, the numerical estimation of the eight free parameters in equation (2). This is a non-linear fitting problem, for equation (2) is non-linear in some of the parameters (particularly the periods). Thus we implemented the non-linear least-squares estimator, called "CURFIT", according to Bevington [1969, pp.232-242]. It uses the Marquardt algorithm which 
combines a gradient search with an analytical solution developed from linearizing the fitting function. This algorithm is found to be robust and fast.

\subsection{Extrapolation and Error Analysis}

As an experiment, we now apply the floating-period estimator described above to the $\mathrm{X}$ - and Y-series of a homogeneous BIH polar motion data set, given at 5-day intervals spanning 1967.01983.3 with the origin adjusted to the CIO in 1967 [Feissel, 1980]. These two time series, henceforth called BIH-X(t) and BIH-Y(t) respectively, are referred to the 1980 Nutation Theory [Capitaine \& Feissel, 1983], and have been smoothed by means of the Vondrak algorithm. They are displayed in Figure 1.

Our basic procedure is straightforward. We simply "chop" an $\mathrm{N}$-year segment (where $\mathrm{N}=5$, $6,7,8$ ) from BIH-X (or BIH-Y), apply the floating-period estimator, and use the derived model parameters to extrapolate the time series $M$ days (where $M=25,50,100,180,365$ ) into the future. We start from the first $\mathrm{N}$-year segment at the beginning of $\mathrm{BIH}-\mathrm{X}$ (or BIH-Y), make an M-day prediction, and then shift forward $\mathrm{M}$ days for the next M-day prediction. This procedure continues across the length of BIH-X (or BIH-Y) until we hit the end. Each M-day prediction can now be compared with actual observations, and the root-mean-square (rms) prediction error (for each M-day prediction), denoted by $E$, can be evaluated. Finally, we can average the sequence of the prediction errors $E$ obtained consecutively across the whole length of BIH-X (or BIH-Y) to yield the average prediction error $\bar{E}$. The value of $\bar{E}$ will be used as a performance indicator for the predictor under consideration. The prediction scheme described above will be denoted as [N(years), $M$ (days)], where $N$ is referred to as the base length and $M$ the prediction length; and we shall be speaking of $E[N, M]$ (a sequence) and of $\bar{E}[N, M]$ (a single quantity).

Figure 2 summarizes the performance of our floating-period predictor by showing $\bar{E}[N, M]$ for $\mathrm{N}=5 \sim 8$ (years) and $\mathrm{M}=25 \sim 365$ (days). Two things are to be noted:

(i) Minimum $\bar{E}$ invariably occurs around $N=6$ (years). This is in fact expected, because 6 years is the beating period between the annual and the Chandler wobbles. For $\mathrm{N}<6$, the presence of 


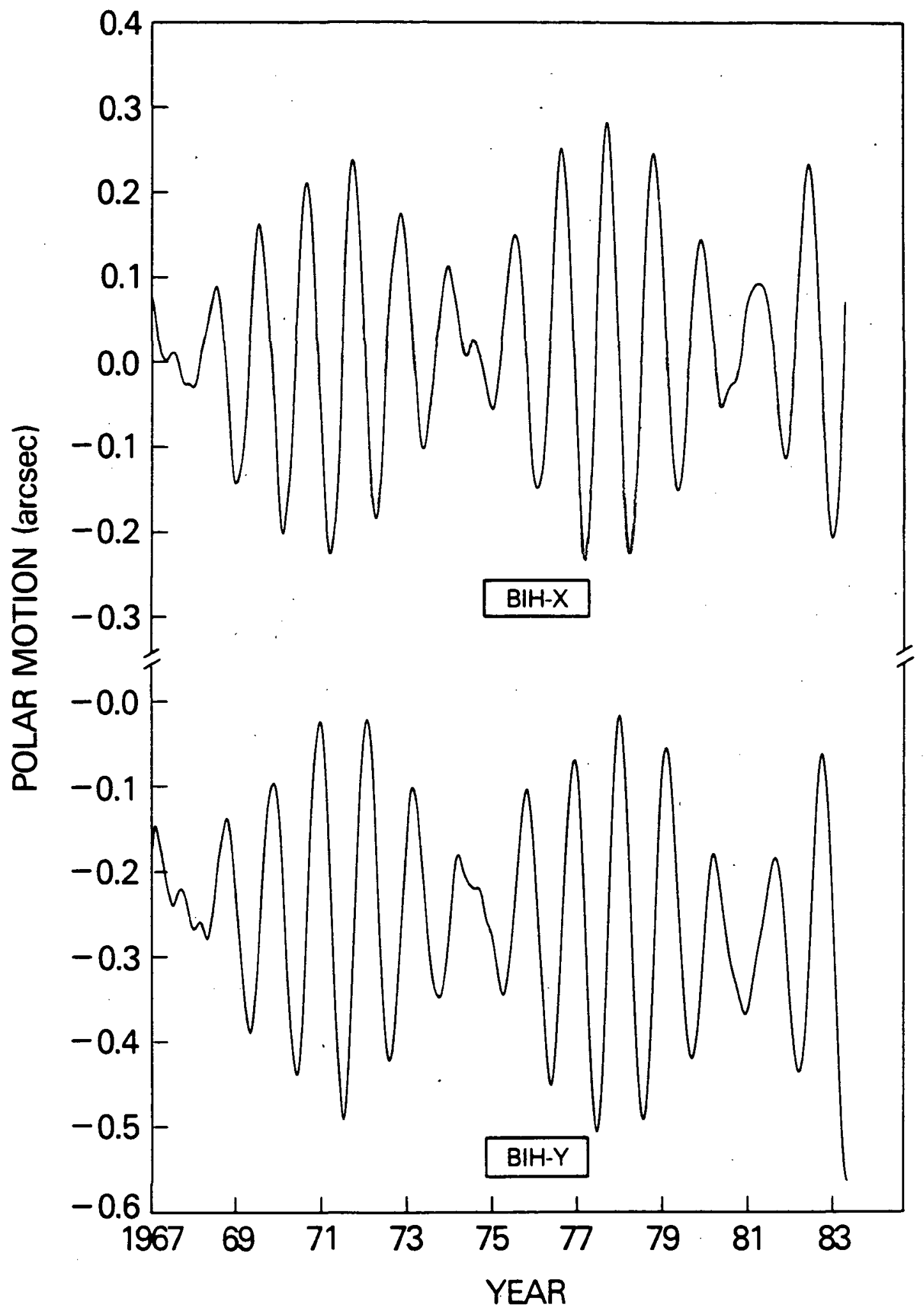

Figure 1. BIH polar motion time series, BIH-X and BIH-Y. 
noise in the data greatly decreases the capability of our floating-period estimator (or any other estimator for that matter) in resolving the two wobbles. (Note, incidentally, that all we need to estimate the set of 8 parameters are 8 data points, which in the present case amount to a base length of only 40 days, if the data were noise-free.) For $N>6$, on the other hand, $\bar{E}$ increases because the coherency of each component in the polar motion decreases as time span gets large; and Figure 2 clearly implies that the coherence length of the polar motion is less than 6 years. Thus, we conclude that 6 years is the optimum base length for the prediction of polar motions.

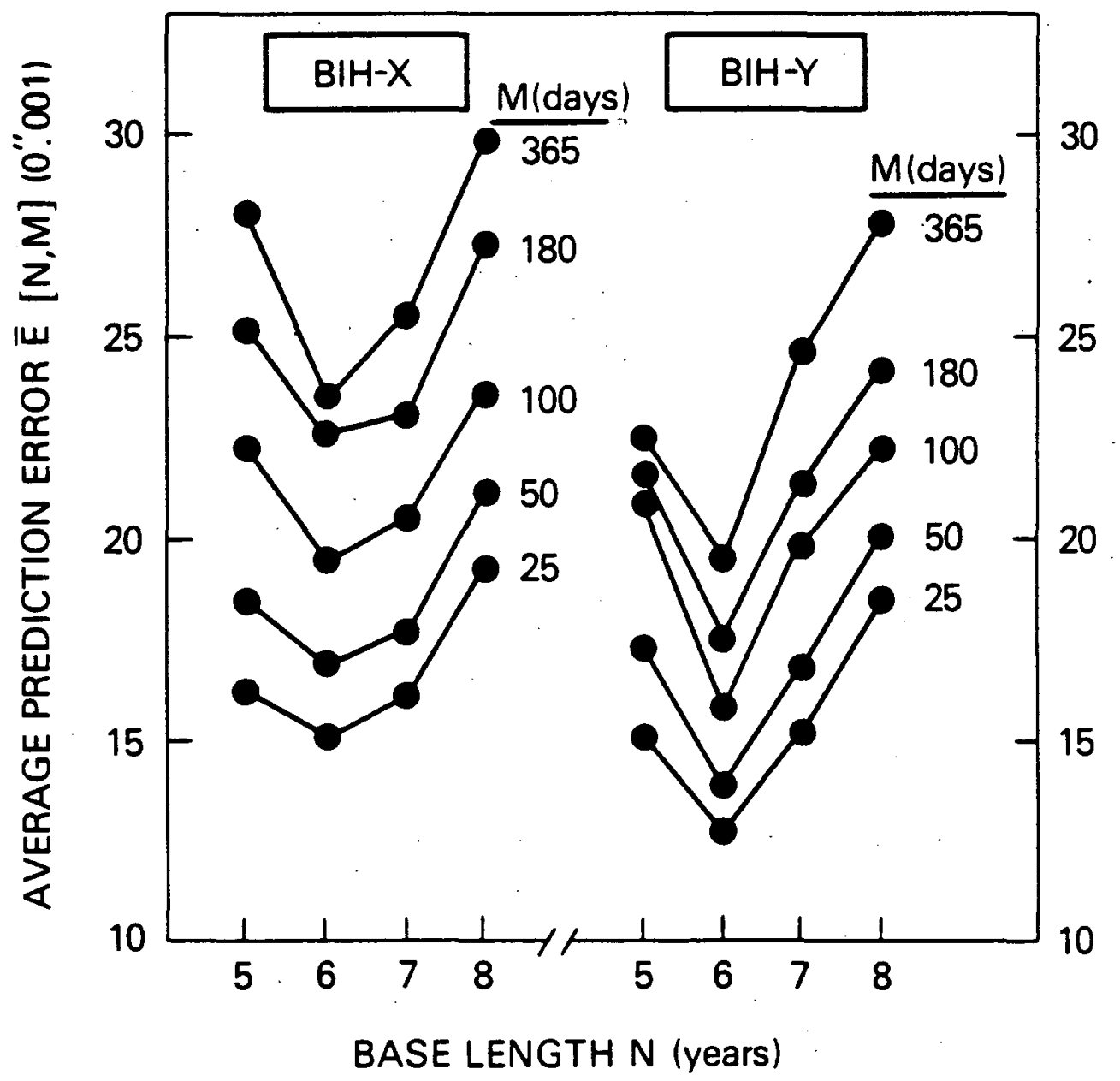

Figure 2. The average prediction error $\bar{E}[N, M]$ by the floating-period predictor, where $N$ is the base length (in years) and $M$ is the prediction length (in days). 
(ii) For any base length $\mathrm{N}, \overline{\mathrm{E}}$ increases monotonously as the prediction length $\mathrm{M}$ gets longer.

This is again to be expected because of the decaying of the coherence, and hence predictability, of the polar motion with respect to time.

As an example, we plot in Figure 3 a typical 3-year segment (1977.7-1980.7) of observed pole positions (solid line) compared with those predicted by some ten consecutive $[6,100]$ floatingperiod predictions (dotted line, at 5-day in tervals). The two sequences $E[6,100]$ (for BIH-X and $\mathrm{BIH}-\mathrm{Y}$ ) are shown as the dashed lines in Figure 4 (their averages, $\bar{E}[6,100]$, are shown in Figure 2 as well as in Table 1). Table 1 lists $\bar{E}[6, M]$ vs. $M$, and is plotted in Figure 5 as dashed lines. From Table 1 we see that, depending on the prediction length (within $25 \sim 365$ days), the floating-period predictor in general achieves accuracy to within $0^{\prime \prime} .012$ to $0^{\prime \prime} .024$. In comparison, the 6-year rms fit residuals of the floating-period estimator are in the range $0^{\prime \prime} .011-0 " .014$.

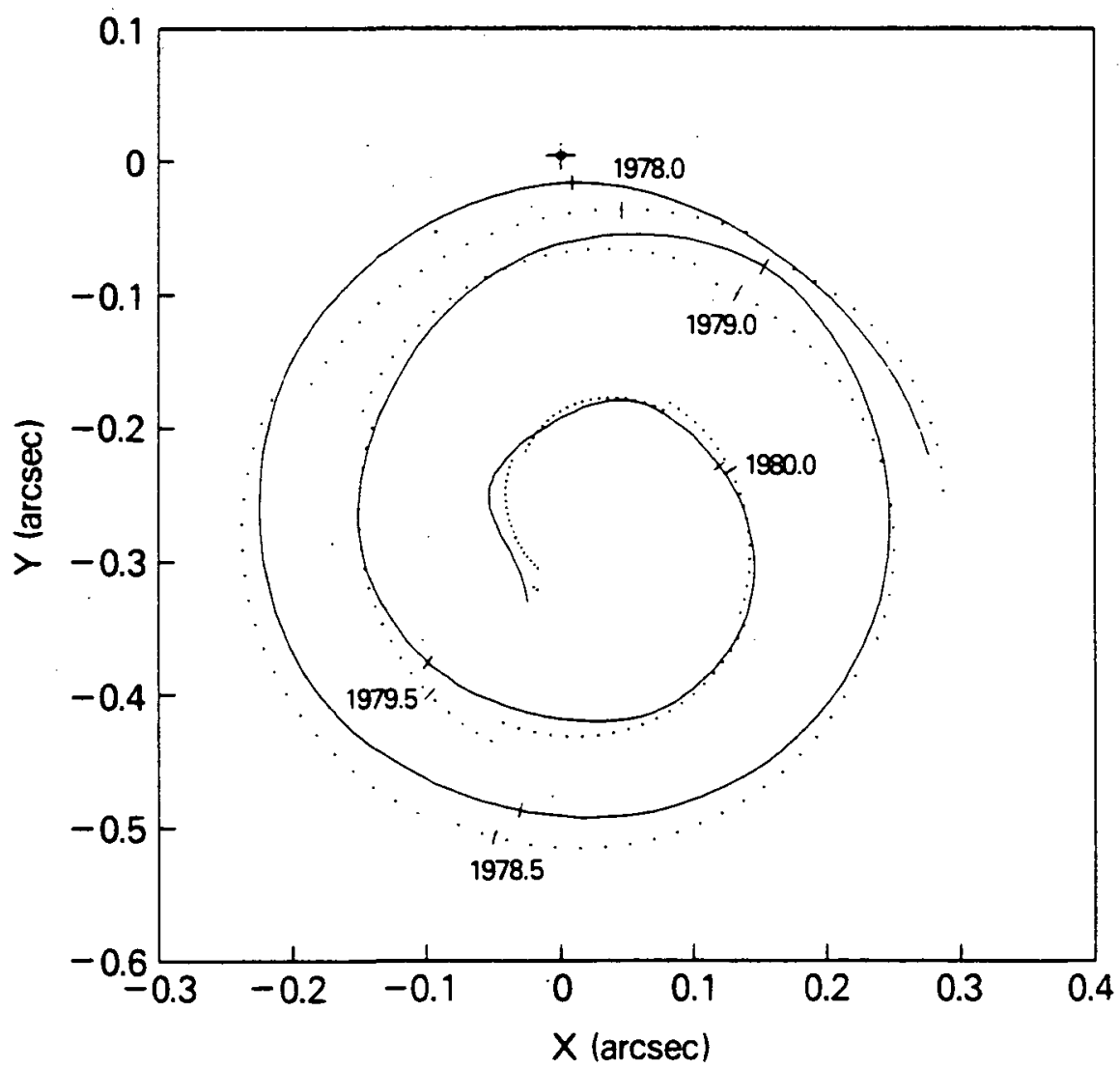

Figure 3. A typical 3-year segment of the polar motion. The solid line is the BIH pole path, the dotted line shows the pole path predicted by the floating-period $[6,100]$ scheme. The cross + gives the position of the 1967 CIO. 
Table 1

The average prediction error $E[6, M]$ (in unit of $0^{\prime \prime} .001$ ) by the floating-period predictor, vs. the prediction length $M$ (see dashed lines in Fig 5).

\begin{tabular}{l|ccccc} 
M (days) & 25 & 50 & 100 & 180 & 365 \\
\hline BIH-X & 15.1 & 16.9 & 19.6 & 22.6 & 23.6 \\
BIH-Y & 12.8 & 13.9 & 15.8 & 17.5 & 19.5
\end{tabular}

\section{OTHER PREDICTORS}

In this section we shall compare other predictors which, when subjected to the same experiment described in Section 2.3, give less satisfactory performance than the floating-period predictor.

\subsection{Fixed-Period predictor}

Fixed-period predictor is one that uses pre-determined annual and Chandler wobble periods in the numerical model, hence the name "fixed-period". Let the periods be 365.25 and 435.00 days, respectively:

$$
X(t)=A+B t+C_{a} \cos \left(\frac{2 \pi t}{365.25}+\phi_{a}\right)+C_{c} \cos \left(\frac{2 \pi t}{435.00}+\phi_{c}\right)
$$

and similarly for $\mathrm{Y}(\mathrm{t})$. This is the simplest polar motion model, and the estimation of the six parameters is a linear problem (the non-linearity with respect to $\phi$ in expression (3) dissolves when we expand the cosine terms). To date, the fixed-period predictor has been the only predictor in use [BIH Rapid Service, USNO Series 7], or discussed [Zhu, 1982] .

However, when we apply the fixed-period predictor to the BIH data set, something unexpected appears. The solid lines in Figure 4 show the prediction error sequence $E[6,100]$ by the fixedperiod predictor for BIH-X and BIH-Y. Prior to the year 1980, this predictor worked steadily, although not quite as well as the floating-period predictor. For example, E[6,100] prior to 1980 is found to be $0 " .021$ for BIH-X and $0 " .018$ for BIH-Y. This agrees with the results of Zhu [1982], and is about $10 \%$ higher than those of the floating-period predictor (c.f. Table 1). However, $\mathrm{E}[6,100]$ from the fixed-period predictor soars to an average level of $0^{\prime \prime} .070$ for BIH-X and $0^{\prime \prime} .059$ for BIH-Y after 1980 (see solid lines in Figure 4). The same phenomenon occurs in all other pre- 
diction schemes $[6, M]$ with different prediction lengths $M$. The root of this phenomenon is traced to a dramatic decrease in both annual and Chandler wobble periods starting around 1977 (see Section 4), a situation that the fixed-period predictor cannot cope. This is also reflected in a similar post-1980 jump in the 6-year fit residuals by the fixed-period estimator.

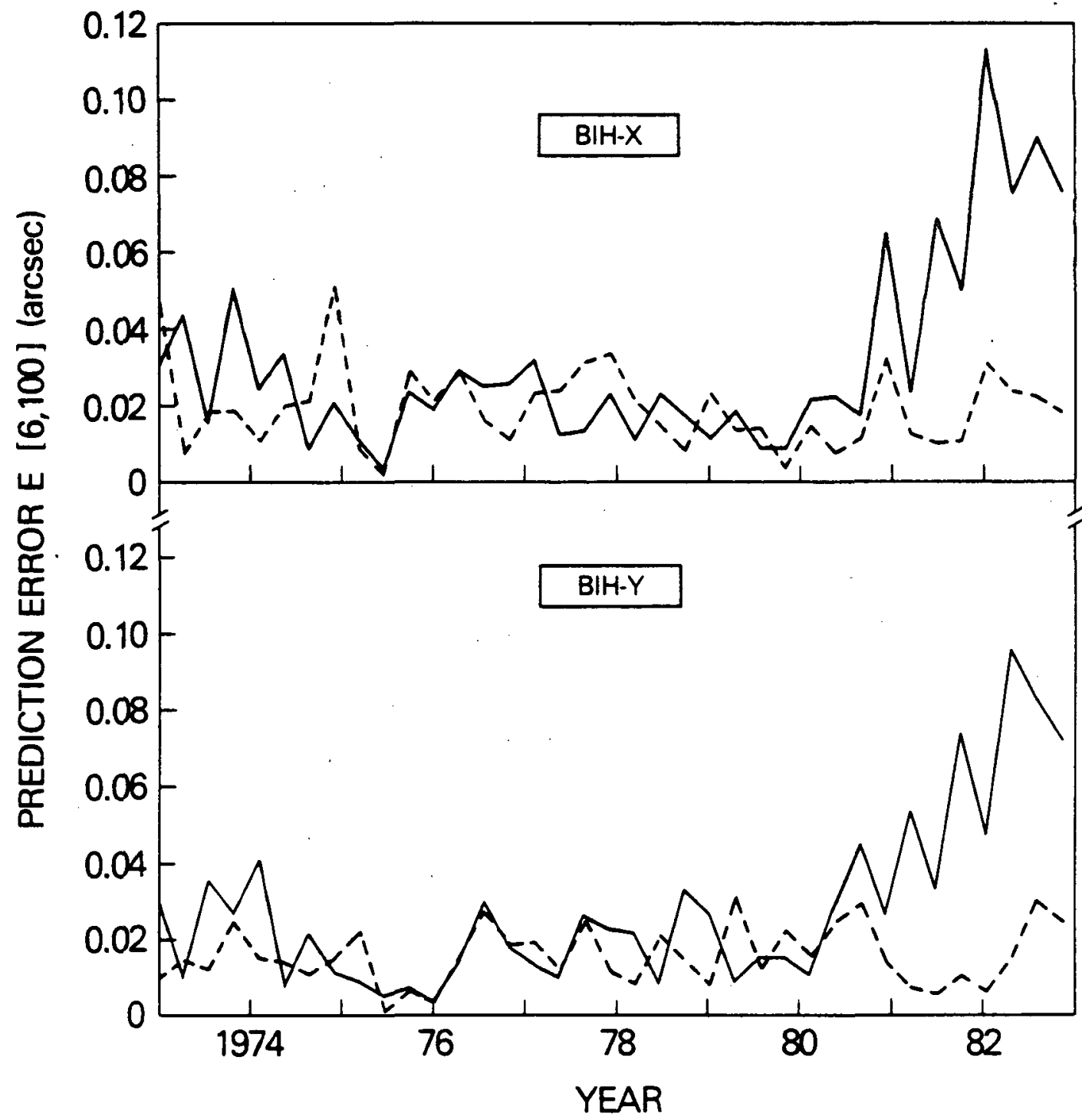

Figure4. Comparison of the prediction error sequence $E[6,100]$ by the fixed-period predictor (solid lines) and by the floating-period predictor (dashed lines). 
The U.S. Naval Observatory (USNO), in its weekly "Time Service Announcement Series 7 (Earth Orientation Bulletin)", publishes 40 days in advance pole positions predicted by a fixedperiod predictor. The major difference between the USNO scheme and the fixed-period scheme discussed above is in the base length used in prediction - USNO uses a base length of only 2 years [McCarthy, personal communication, 1983]. The advantage of using a short base length is that the resultant estimates are more "instantaneous". In fact; our experiments have confirmed USNO Series 7 by showing that the fixed-period $\bar{E}[2,40]$ lies as low as $0^{\prime \prime} .015$, which is comparable to $\bar{E}[6,40]$ of our floating-period predictor (see the dashed lines in Figure 5). However, this success is only apparent. Indeed, in the fixed-period $[2, \mathrm{M}]$ scheme, since the base length falls much shorter than 6 years, the estimates are prone to noise in the data (see Section 2.3). In particular, this is found to result in wobble amplitude estimates that are unstable and sometimes unreasonable. For short prediction lengths ( $M$ shorter than 40 days, say) this creates little discomfort as far as the prediction is concerned because of the general compensating capability of least-squares estimators. However, when one extrapolates these (unstable) estimates further into the future, one would expect a rapidly growing deviation of the predictions from actual observations. This is indeed found to be the case - for example, $\bar{E}[2,365]$ is found to be $0^{\prime \prime} .029$, almost double the value of $\bar{E}[2,40]$. Furthermore, although not as severely as discussed earlier, the fixed-period $[2, \mathrm{M}]$ scheme also sees a post-1980 jump in $E[2, M]$ as $M$ increases.

In conclusion, the fixed-period predictor yields parameters that fail to reflect true variations in the polar motion; and, as a result, performs less satisfactorily than the floating-period predictor and is potentially dangerous in the sense that it may sometimes lead to disastrous predictions.

\subsection{Floating-Complex Period Predictor}

The superiority of the floating-period predictor to the fixed-period predictor is obviously the consequence of two additional degrees of freedom in the former fitting procedure, namely the two wobble periods. Similarly, we can build in yet more degrees of freedom to allow for variations in 
the amplitude for each $\mathrm{N}$-year segment. A natural choice is to make the wobble periods complex in the numerical model, so that their imaginary parts give exponential decay or growth in the wobble amplitudes, hence the name "floating-complex period":

$$
X(t)=A+B t+C_{a} \exp \left(\alpha_{a} t\right) \cos \left(\frac{2 \pi t}{P_{a}}+\phi_{a}\right)+C_{c} \exp \left(\alpha_{c} t\right) \cos \left(\frac{2 \pi t}{P_{c}}+\phi_{c}\right)
$$

and similarly for $\mathrm{Y}(\mathrm{t})$. Now we have ten (real) parameters to be estimated, some of which are nonlinear; and again we use the "CURFIT" algorithm as in Section 2.2

As expected, equation (4) is indeed found to be a better fitting model. For instance, the rms fit residual for each 6-year record segment.is invariably lower (in some cases as much as 30\%) than that of the floating-period estimator. As a predictor, like the floating-period predictor, it also responds to the post-1977 drop in the wobble periods mentioned in Section 3.1 and shows no increase in the prediction errors $E[6, M]$ after 1980 as did the fixed-period predictor. However, the average prediction error $\bar{E}[6, M](\dot{M}=25 \sim 365)$ of its predicted pole positions (by means of extrapolation) are generally higher than those by the floating-period predictor, as shown in Figure 5 . This, of course, is indicative of the fact that the exponential decay/growth trend in the wobble amplitudes in general does not continue past, say, 6 years. Indeed, physically, we should not expect otherwise because the variations of wobble amplitude with respect to time are determined by the excitation functions which presumably are either erractic or changing from year to year. In conclusion, we state that while the floating-complex period model does a good fitting job and may hence have more potential usages, it is less satisfactory as a predictor for the polar motion. 


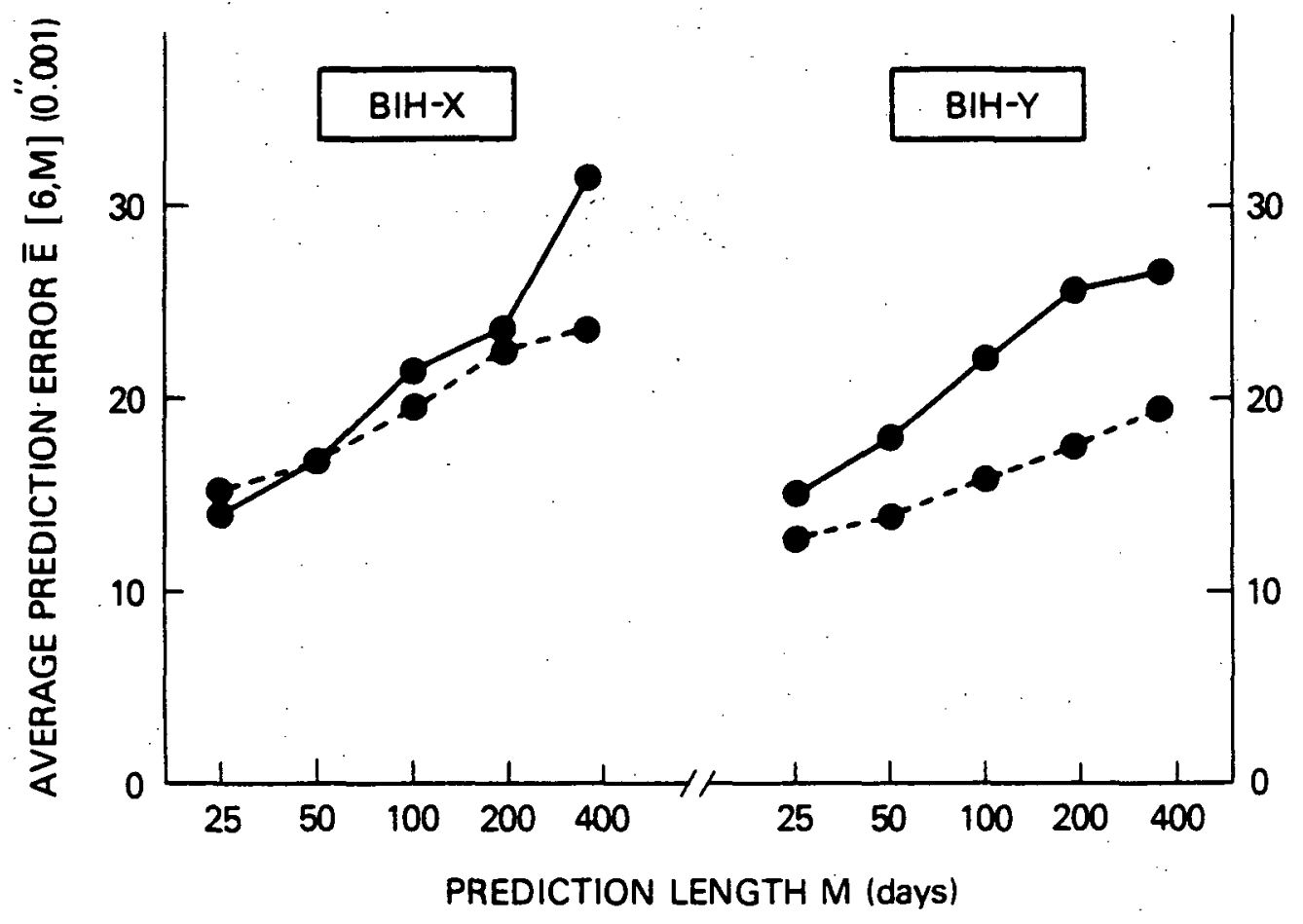

Figure 5. Comparison of the average prediction error $\bar{E}[6, M]$ (as a function of the prediction length $M$ ) by the floating-complex period predictor (solid lines) and by the floatingperiod predictor (dashed lines, see Table 1).

\subsection{Autoregressive (AR) Predictor}

A related prediction scheme (but under a completely different philosophy) is the so-called linear prediction, which has been widely pursued in research fields such as geophysics, speech processing, control theory, and economics [see e.g. Makhoul, 1975]: Its application in time-series prediction has been thoroughly studied by Box \& Jenkins [1970]. Here we shall briefly develop one widely used class of numerical models in the linear prediction theory, namely the autoregressive (AR) models, and relate it to the present problem of polar motion prediction.

An mth order AR model of a time series $x(n)$ is

$$
x(n)=\sum_{i=1}^{m} S_{i} x(n-i)+a(n)
$$

where $\{a(n)\}$ is a random (or white) series, and $\left\{S_{i} ; i=1,2, \ldots, m\right\}$, called the AR coefficients, are 
the (real) model parameters yet to be determined. While having the physical meaning of being the external disturbances to the dynamic system, the series $a(n)$ is just the nth prediction residual as far as prediction is concerned [for details see Box \& Jenkins, 1970]. Many physical systems can be modeled by such an AR process; and the model identification problem consists mainly in the determination of the order $\mathrm{m}$. Although the latter problem may often be different or even controversial, we shall show presently that it poses no obstacle to the modeling of polar motion time series. It is well-known that a pure sinusoidal function can be represented as a second-order AR time series [see e.g. Yule, 1927]. In fact, it can be shown that a linear combination of $\mathrm{K}$ complex sinusoids (with exponentially decaying or growing amplitudes) can be expressed as a $2 \mathrm{Kth}$ order $\mathrm{AR}$ time series, and the $\mathrm{K}$ complex periods are related to the $2 \mathrm{~K}$ (real) AR coefficients through the Prony's relation [for details see Chao \& Gilbert, 1980; Chao, 1984]. For example, with a(n) representing some random excitation function and $m=2$, equation $(5)$ is a mathematically simple, and physically sound model for the Chandler wobble. For a linear combination of two wobbles $(\mathrm{K}=2)$, namely the annual and the Chandler wobbles in the polar motion, we let $\mathrm{m}=4$. Equation (5) is then a general AR model which, apart from the secular term $\mathrm{A}+\mathrm{Bt}$, encompasses equation (2), (3), and (4).

Now that the numerical model has been built, the estimation of parameters (i.e., the AR coefficients $\left.\left\{S_{i} ; i=1,2,3,4\right\}\right)$ is just a linear least-squares procedure, and the extrapolation is straightforward, all according to equation (5) by letting $a(n)=0$. In practice, unfortunately, we encounter the following two difficulties:

(i) Since equation (5) does not allow for a linear term $\mathrm{A}+\mathrm{Bt}$, the latter has to be removed from the data prior to the AR estimation. This can be done by a least-squares fit. However, for record segments as short as several years, the fit is biased in the presence of the wobble terms. Synthetic experiments have indicated that this, in turn, can lead to biased estimations for the wobble parameters. (Nevertheless, it should be pointed out that the AR estimator is accurate 
and powerful in analyzing zero-mean, trendless polar motion data, as demonstrated by Chao [1983]).

(ii) A more serious problem which is inherent in the AR predictor results from the noise in the data. Thus, even with an accurate set of model parameters, a predicted value can be, and often is, seriously biased by the noise in the existing values used in the prediction. Put differently, the noise in $x(n-i)$ on the right side of equation (5) "propagates out" to $x(n)$ on the left side of (5). Synthetic experiments show that average prediction errors E generated by an AR predictor are generally several times larger than the corresponding E's by a floating-period predictor.

Thus, although it is an adequate representation for the wobbles, the AR model (5), and hence the AR predictor, is unworthy in the prediction of polar motions.

\section{CONCLUSIONS AND DISCUSSIONS}

As Zhu [1982] pointed out, the prediction errors consist of modeling errors and observation errors in the data. Assume that the modeling error and the observation error in both $\mathrm{X}$ - and $\mathrm{Y}$ components are uncorrelated with one another. Then the sum of their individual variances equals the variance of the total prediction error:

$$
\sigma_{\mathrm{E}}^{2}=\sigma_{\mathrm{M} . \mathrm{E} .}^{2}+\sigma_{\mathrm{O} . \mathrm{E} .}^{2}
$$

where each term is in turn the sum of the variances for X-and Y-components ( $\sigma$ denotes the standard deviation). Now let us define the predictability P simply as

$$
\mathrm{P}=1-\sigma_{\text {M.E. }} / \sigma_{\text {P.M. }}
$$

where $\sigma_{P . M}$ is the standard deviation of the polar motion. For a system being modeled reasonably we expect $\mathrm{P}$ to lie between 0 and $1 ; \mathrm{P}=1$ signifies a completely deterministic system which has been modeled exactly, while $\mathrm{P}=0$ results from a complete failure in prediction with the modeling error as great as the signal itself. Obviously $\mathrm{P}$ depends on the predictor and the prediction 
scheme in use, and represents a (linear) performance indicator. The quantity $\sigma_{\text {P.M. }}$. can be estimated by computing the rms amplitude of the polar motion time series, and is found to be 0 ". 17 . The quantity $\sigma_{M . E .}$ can be determined using equation (6), where (i), $\sigma_{O . E .}$ is estimated to be about $0 " .011$ for our smoothed BIH data set [Feissel, personal communication, 1983], and (ii) $\sigma_{E}$ can be estimated simply using $\bar{E}$, an essemble average of $E$ which is itself an estimate (as a temporal mean) of $\sigma_{E}$. Thus, from Table 1 and equation (7), we conclude that the predictability of the polar motion using the floating-period predictor for prediction lengths in the range $25 \sim 365$ days is $91-83 \%$. The predictability of the polar motion using the floating-complex period predictor is somewhat lower (90-77\%); and those using the fixed-period and AR predictors are considerably lower yet, and, in the former case, sometimes ill-behaved (see Section 3.1).

One obvious by-product of our prediction procedure is the set of sequences of estimated polar motion parameters as functions of time. One such sequence which is interesting and relevant to our present study is that of the estimated wobble periods. Figure 6 shows the variations in the estimated annual and Chandler wobble periods resulted from the $[6,100]$ prediction scheme. The points represent 6-year running estimates of the periods, each shifted 100 days down the length of the BIH data set; thus features with scale shorter than, say, 2 years might be spurious (being simply the results of noise in the data). However, the sudden decrease starting around 1977 in both annual and Chandler wobble periods, which show up in both BIH-X (solid lines) and BIH-Y (dashed lines). is believed to be real. Other estimation techniques, namely the floating-complex period and the AR estimators, also unequivocally show a very similar trend in the period variations. This has caused our implementation of the fixed-period predictor to "fail" after 1980 as we have seen in Section 3.1. Presently we know nothing about its cause(s) or its geophysical implications. We can only speculate that this phenomenon has its root in the atmospheric circulation system; but this awaits further investigations. 


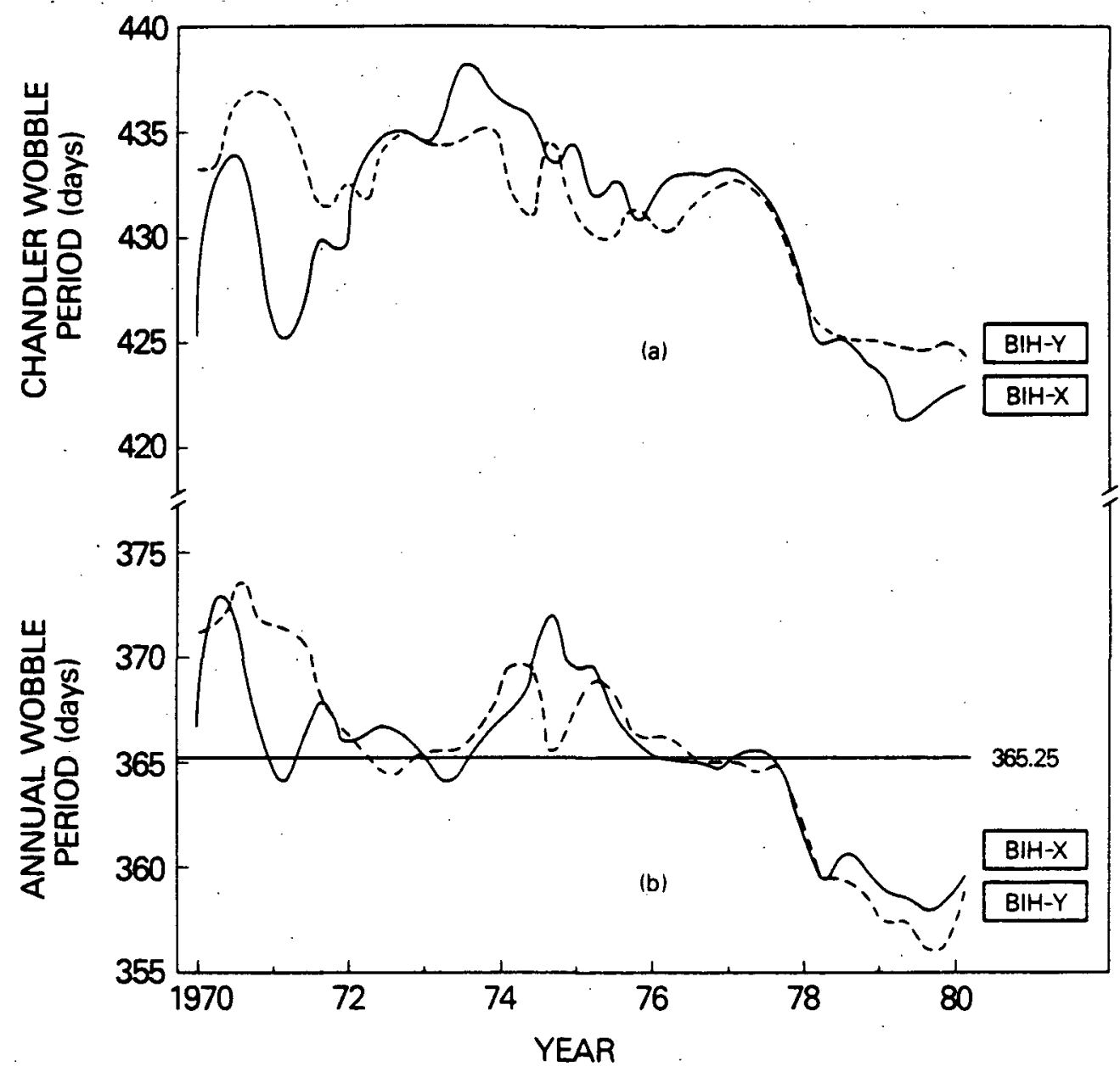

Figure 6. Six-year running estimates of wobble periods estimated by the floating-period estimator from BIH-X (solid lines) and BIH-Y (dashed lines) for (a) the Chandler wobble, and (b) the annual wobble. The curves have been connected smoothly. 


\section{REFERENCES}

Babcock, A. K., Predicting Earth's rotation, EOS, Trans. Amer. Geophys. Union, 63, 903, 1982.

Bevington, P. R., Data Reduction and Error Analysis for the Physical Sciences, McGraw-Hill, New York, pp. 336, 1969.

Box, G. E. P., and G. M. Jenkins, Time Series Analysis: Forecasting and Control, Holden-Day, San Francisco, 1970.

Capitaine, N., and N. Feissel, the introduction of the IAU 1980 Nutation Theory in the computation of the Earth rotation parameters by the Bureau International de l'Heure, Bull. Geodesique, 57 , 198-204, 1983.

Chao, B. F., Autoregressive harmonic analysis of the Earth's polar motion using homogeneous ILS data, J. Geophys. Res., 88, 10299-10307, 1983.

Chao, B. F., On the maximum entrophy/autoregressive modeling of time series, NASA TM 86057 , 1984.

Chao, B. F., and F. Gilbert, Autoregressive estimation of complex eigenfrequencies in low frequency power spectra, Geophys. J. Roy. Astron. Soc., 63, 641-657, 1980.

Colombo, G., and I. I. Shapiro, Theoretical model for the Chandler wobble, Nature, 217, 156-157, 1968.

Feissel, M., Determination of the Earth rotation parameters by the Bureau International de l'Heure: 1962-1979, Bull. Geodesique, 54, 81-102, 1980.

Lambeck, K., The Earth's Variable Rotation: Geophysical Causes and Consequences, Cambridge Univ. Press, New York, 1980.

Makhoul, J., Linear prediction: a tutorial review, Proc. IEEE, 63, 561-580, 1975.

Yule, G. U., On a method of investigating periodicities in disturbed series with special reference to Wolfer's sunspot numbers, Phil. Trans. Roy. Soc., A, 226, 267-298, 1927.

Zhu, S. Y., Prediction of polar motion, Bull. Geodesique, 56, 258-273, 1982. 
BIBLIOGRAPHIC DATA SHEET

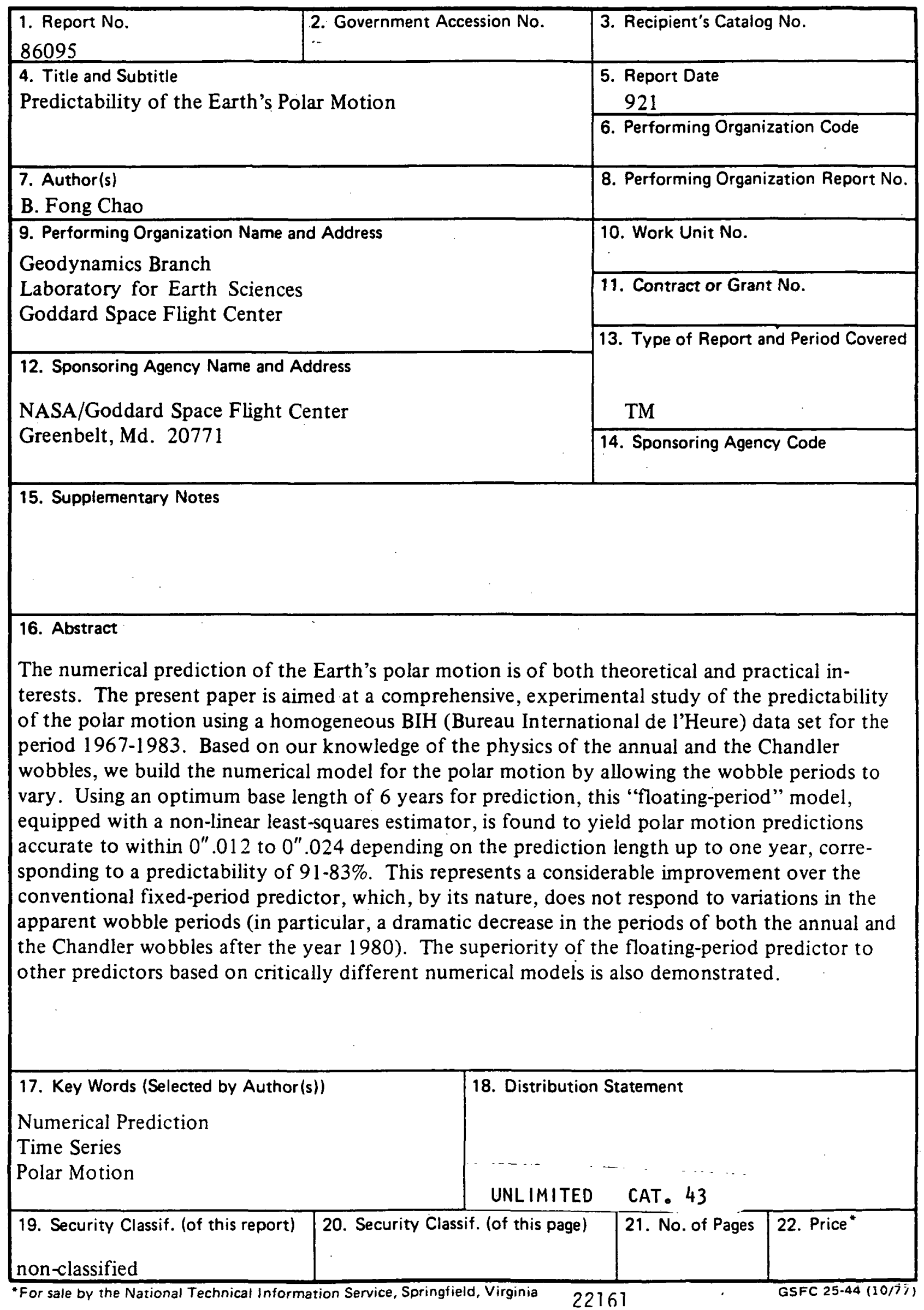

